\title{
WHAT IS A GOOD MIDWIFE? \\ INSIGHTS FROM THE LITERATURE.
}

\author{
Sara E. Borrelli
}

\begin{abstract}
Objective. To review the literature around what is considered to be a good midwife and in particular what women value in a midwife, in order to identify the gaps in the evidence for future research. Design. This paper reviews the research in the area of interest over the past 30 years. The literature search focused on the concept of good midwife using synonyms and antonyms. The inclusion criteria included language (English or Italian). The examined databases were Medline, Maternity and Infant Care, Applied Social Sciences Index and Abstract and CINAHL. Setting. Studies conducted in high-income countries were taken into account. A focused review of papers which explicitly investigated what a good midwife means and a thematic analysis on what women value in a midwife were carried out. Participants. Different standpoints have been considered (midwives, student midwives, women and their partners), focusing in particular on women viewpoint. Findings. The literature review reveals information about what is considered to be a good midwife from a range of perspectives and what women value in a midwife. A good midwife should possess several attributes: theoretical knowledge, professional competencies, personal qualities, communication skills and moral/ethical values. According to the thematic analysis around what childbearing women value in a midwife, frequent key-themes emerging from the literature were: support, possibility of choice, feeling in control and having appropriate information. Key conclusions. The meaning of good midwife might change according to different actors involved in midwifery care and there is no agreement on the definition of what constitutes a good midwife. Furthermore, it is not clear if what women value in a good midwife corresponds to the midwives' perception of themselves as good professionals. There is a dearth of information around women's expectations and experiences specifically of a good midwife, and even less around whether this changes according to where they give birth. Implications for practice. This literature review seeks to stimulate debate and reflection among midwives and professionals involved in the childbearing event, in order to fulfil women's expectations of their midwife and increase their satisfaction with the birth experience. The identification of the gaps in the evidence provided the starting point and allowed the development of research questions and methodology for an ongoing doctoral research. Based on the gaps in the evidence, the doctoral research will explore and seek to explain nulliparous women's expectations and experiences of a good midwife in the context of different planned place of birth, using a Grounded Theory methodology. It is also expected that the findings of this literature review will stimulate additional research in this area to ultimately inform midwifery practice and midwifery educational programmes.
\end{abstract}

KEY WORDS: good midwife; literature review; thematic analysis; childbearing woman/women; support; choice; control; information. 


\section{Introduction}

This paper reports the literature review around what is considered to be a good midwife and in particular what women value in a midwife, in order to identify the gaps in the evidence for future research. The author's personal interest in what is considered to be a good midwife comes mainly from experience in midwifery practice, the debates and discussions with colleagues around the topic and reflection on midwives' behaviors in their everyday practice. In fact, maternity services are sometimes characterised by a lack of caring for women and their families. Short-staffing, busyness and medicalised models of care may contribute to this perception (Larkin et al., 2012). Reflecting on professional values, attitudes, competencies and quality of midwifery care, a fundamental question arises: what is a good midwife. The answer is probably ambiguous, since it could have different meanings and interpretations, related to who answers the question or when and where it is answered.

The word midwife is literally translated with woman. A regulatory definition of the midwife is endorsed by the International Confederation of Midwives (ICM), the World Health Organization (WHO) and the International Federation of Gynecology and Obstetrics (FIGO): 'the midwife is recognised as a responsible and accountable professional who works in partnership with women to give the necessary support, care and advice during pregnancy, labour and the postpartum period, to conduct births on the midwife's own responsibility and to provide care for the newborn and the infant'. In particular, the International Confederation of Midwives (Fullerton et al., 2011) delineates the knowledge, skills and behavior that would characterize the domain of competencies of the midwife who is educated according to the international definition of the profession. 
In trying to answer the question of what constitutes a good midwife, it has to be acknowledged that the word good could have different meanings and understandings. In fact, it can be defined in several ways, depending on the context and the subject or object it is referred to. The Oxford Dictionary (online) defines good as follows: to be desired or approved of; having the required qualities; of a high standard; possessing or displaying moral virtue; giving pleasure; enjoyable or satisfying; thorough; valid. However, the definition of a good midwife as one with satisfactory enough theoretical knowledge and practical skills guarantees a minimum standard but does not encourage excellence: in the sense that the professional is required to perform to a proficient minimum. From this perspective, personal attitudes seem to be irrelevant and what is really important is the absence of incompetence instead of the possession of excellence, forgetting other essential characteristics of midwifery such as personal qualities and moral/ethical values (Sellman, 2007).

The International Confederation of Midwives (ICM) supports, represents and works to strengthen professional associations of midwives throughout the world. The ICM works with midwives and midwifery associations globally to secure women's right and access to midwifery care before, during and after childbirth. Essential Competencies for Basic Midwifery Practice were published in 2010. The key midwifery concepts that define the unique role of midwives in promoting the health of women and childbearing families have been identified as follows: partnership with women; respect for human dignity and rights; advocacy for women; cultural sensitivity; focus on health promotion and disease prevention that views pregnancy as a normal life event.

As the doctoral study which will follow this literature review will take place in UK, what the Nursing and Midwifery Council (NMC) says about good midwifery practice has been taken into account. The NMC is a regulatory body that regulates nurses and 
midwives in England, Wales, Scotland, Northern Ireland and the Islands. The Nursing and Midwifery Council sets standards of behaviors, performances and ethics for midwives. Following this code of conduct (last version 2008), the midwives that meet these standards are expected to give high quality care throughout their professional life. In fact, the code is central to good midwifery, and all midwives must follow it in order to be able to work legally and safely. The four central principles of the code are: individualised care and respect for dignity; multidisciplinary work and promotion of health; high standards of practice and care; being open and honest with integrity and upholding the reputation of the profession. Any midwife must meet these standards and regularly demonstrate that they are meeting them, in order to remain on the register and be eligible to work in the UK.

Neither the ICM nor the NMC speak specifically about the notion of good midwife. In fact, they refer only to the term midwife, identifying skills and competencies required of them at the point of registration and throughout their professional career. In addition to this, the standards of ICM and code of ethics of NMC have been entirely developed by professionals. However, the needs of women might not match with the midwives' perceptions of themselves as good caregivers. This issue will be addressed later in this paper.

\section{Methods}

This paper reviews the research in the area of interest over the past 30 years. The literature search focused on the concept of good midwife using synonyms and antonyms as key words. Different standpoints have been considered (midwives, student midwives, women and their partners). The inclusion criteria included language 
(English or Italian - the author is Italian). Studies conducted in high-income countries were taken into account. The examined databases were Medline, Maternity and Infant Care, Applied Social Sciences Index and Abstract and CINAHL.

A focused review of papers which explicitly investigated what a good midwife means will be presented. In order to focus on the specific definition of what a good midwife is, a selection of papers was reviewed. The inclusion criterion was the use of the terms good midwife or good midwifery in the title and/or in the aim of papers. Only six papers explicitly investigated what a good midwife means, including an integrative review (Nicholls \& Webb, 2006), a Delphi study (Nicholls et al., 2011), an evolving theory (Halldorsdottir \& Karlsdottir, 2011) and three qualitative researches (Byrom \& Downe, 2010; Carolan, 2010; Carolan, 2012).

A rationale will be given for focusing on women's standpoint and a thematic analysis on what childbearing women value in a midwife during labour and birth will be included. The papers were selected from the body of literature identified in the literature search around the concept of good midwife. The inclusion of the studies in the thematic analysis was done on the basis of the methodological approach (inclusion criteria: empirical research), the sample (inclusion criteria: women as participants) and pragmatic reasons (inclusion criteria: focus of the study totally or partially related to the women's views of the midwife during labour and birth). Only studies conducted in high-income countries were examined. Time limits and sample size were not considered as criteria for the selection of the studies. Six papers have been included in the thematic analysis. The studies were conducted over a 23 years period (from 1987 to 2010 ) and the sample sizes vary from eight to 825 women. Women's partners are involved in two of the selected studies. Methodologies were variously described as qualitative ( $n=4)$ (Berg et al., 1996; Brown et al., 2009; Dahlen et al., 2010; Walker 
et al., 1995), using a phenomenological $(n=1)$ (Berg et al., 1996) or Grounded Theory approach ( $\mathrm{n}=3$ ) (Brown et al., 2009; Dahlen et al., 2010; Walker et al., 1995). Two authors utilised quantitative methods (Green et al., 1990; Tumblin \& Simkin, 2001). The selected papers for the thematic analysis about what women value in a midwife have been critically appraised by the author through validated tools, in order to increase the methodological strength of this work. The appraisal of the qualitative studies was done using the tool developed by Walsh \& Downe (2005). The quantitative elements of the papers were appraised using the Critical Appraisal Skills Programme tools (Public Health Resource Unit, 2006). Details of the papers and associated critique are reported in Table 1. The selected papers were read several times in order to grasp the essential features and to identify the main themes. Themes recurring in at least three of the total six papers have been considered by the researcher. The contents of the papers have been utilised to describe and explain the themes. It must be acknowledged that the authors of the selected studies had not explicitly asked to women what they consider to be a good midwife. However, they either totally or partially relate to what women value in a midwife. Some references to the papers of the broader body of the literature will be included in the thematic analysis in order to contextualize the recurring themes. Finally, literature gaps in knowledge of the meaning of good midwife will be given.

\section{Findings}

\section{What is a good midwife?}

The term good in relation to midwives' attributes and quality of midwifery practice has been widely debated and researched. According to the literature about this topic, 
different meanings and interpretations are mainly related to the concept of what is a good midwife.

Nicholls \& Webb (2006) aimed at identifying a research-based definition of a good midwife that could be used as an operational definition for the purposes of curriculum development. They carried out an integrative review of methodologically-diverse research papers. Thirty-three research-based papers were included in the review. The authors found that the principal attribute in these papers on being a good midwife was having good communication skills. Being compassionate, kind, supportive (affective domain), knowledgeable (cognitive domain) and skilful (psychomotor domain) also made major contributions. Being involved in education and research were necessary requirements, and midwives' abilities to treat women as individuals, adopting a caring approach and being there for women were essential. Furthermore, the authors argue that a good midwife can compensate for poor management systems. However, the researchers do not distinguish between the different standpoints of midwives, students, women and their partners. Thus, it is not clear if what women value in a good midwife corresponds to the midwives' perception of themselves as good professionals. After noting the lack of studies addressing the overarching question "What makes a good midwife?", Nicholls et al. (2011) investigated the perceptions of the good midwife using a Delphi questionnaire. The sample included postnatal women, midwives and midwifery educators $(n=226)$. The statements with the highest mean scores were related to lifelong learning, woman-centred care and again good communication skills. The authors found that being a lifelong learner and the development of good interpersonal skills are as important as technical competence in making a good midwife. Again, the researchers do not distinguish between women and midwives' 
answers in the sections of findings and discussion of data in this study. Thus, it is difficult to disentangle the participants' accounts.

Halldorsdottir and Karlsdottir (2011) introduced an evolving theory on the empowerment of childbearing women, where the midwife's professionalism is central. The theory is synthesized from nine datasets from nine original research papers of their own studies and scholarly work, and more than 300 studies were reviewed for clarification and confirmation. According to the theory, the professional midwife cares for the childbearing woman and her family and is professionally competent. She/he has professional wisdom and interpersonal competence and is capable of empowering communication and positive partnership with the woman and her family. Furthermore, the professional midwife develops herself both personally and professionally. The author states that this evolving theory must be regularly reviewed both in the light of current midwifery knowledge and conceptions around the idea of who is considered to be a good midwife. However, their theory is limited because it is descriptive rather than explanatory. Furthermore, the identified themes are not comprehensively discussed in the findings.

Considering the standpoint of midwives $(n=10)$, Byrom \& Downe (2010) investigated the characteristics of the good midwife through a phenomenological approach. The data analysis underlined two clear dimensions: skilled competence (knowledge, skills and competencies) and emotional intelligence (personal qualities). The authors used an appreciative inquiry method which deliberately focuses on positive characteristics of the midwife. Thus, it has limited utility in distinguishing good qualities and attributes from poor ones.

Student midwives' views $(\mathrm{n}=32)$ of the good midwife have been explored by Carolan (2010; 2012). Carolan (2010) found that first year students spoke of a series of key 
attributes they felt were important to the role of the midwife: personal qualities and attitudes, belief in women and natural birth, ethical concerns and possession of additional attributes (life experience, cultural knowledge and passion/enthusiasm). Students, early on in their course, showed a clear understanding of the affective attributes required of a good midwife but less understanding of requirements of knowledge and competence. Two years later, Carolan (2012) explored third year midwifery students' view of a good midwife and it was evident that their perceptions were becoming aligned with the views of qualified midwives. In fact, final year's students acknowledged the importance of safe practice at the same time as supporting women to make decisions. The author brings to light the importance of 'early transition and socialization into the profession' (Carolan, 2012: 1).

According to the findings of the studies cited above (and therefore from a range of perspectives) a good midwife should possess several attributes: theoretical knowledge, professional competencies, personal qualities, communication skills and moral/ethical values. However, the meaning of good midwife might change according to the ideas of the different actors involved in midwifery care and there is no agreement on the definition of what constitutes a good midwife. Furthermore, the different standpoints often overlap and it is difficult to make a clear distinction between them, as in the papers of Nicholls and Webb (2006) and Nicholls et al. (2011). As stated above, it is not clear if what women value in a good midwife corresponds to the midwives' perception of themselves as good professionals.

\section{Why focus on the perspective of childbearing women?}

The role of the midwife has undergone a number of changes in recent decades, primarily related to a greater emphasis on woman-centered care, both in policy 
documents (International Confederation of Midwives, 2010; Nursing and Midwifery Council, 2008) and in the research literature (Fontein, 2009; Freeman et al., 2004; Leap, 2000). Woman-centered care is based on the recognition, acknowledgement and respect of the childbearing woman with her distinctive needs, ideas, thoughts, emotions, expectations and wishes about pregnancy, birth and motherhood. In other words, the woman and her baby come first (Fontein, 2009). Green et al (1988) argued that the woman expects to meet a midwife who will care for her as an individual, supporting her instinctive abilities in becoming a mother. The midwife's main aim should be to attend to the expectant mother and her needs, encouraging her through the birth process and understanding her strengths and weaknesses. Eliasson et al. (2008) argue that because giving birth is such a significant event in a woman's life, it could affect future behaviours of the whole family. Furthermore, positive experiences are embedded in the memory if the midwife has been acting in a caring way (Halldorsdottir \& Karlsdottir, 1996). As Waldenstrom (1998) affirms the constant presence of a midwife and good care during birth can increase the woman's satisfaction with her birth experience. Thus, every midwife should consider the importance of the wellbeing and satisfaction of the woman and her family during the childbearing event.

The importance of the woman's perspective is underlined by Pembroke \& Pembroke (2008), arguing that the woman is the principal actor that invites others to be with her as she gives birth. The authors introduce the concept of genuine hospitality, debating the appropriateness of referring to the midwife as a host. In fact, the midwife could be seen primarily as the invited guest to the experience of the woman. There is still a place for the appellation host in relation to the midwife's role: 'the midwife is called upon to mentally establish an open space that will be filled by the woman's needs and 
preferences' (Pembroke \& Pembroke, 2008: 325). Furthermore, it has to be acknowledged that the potential for psychological benefit or damage is present at every birth. Caregivers have a great deal of influence on how each woman will remember her experience. In addition to a safe outcome, the goal of a positive memories should guide the midwife's care (Simkin, 1991).

Maternity services and midwifery programmes sometimes seem more focused in what the institutions and regulatory bodies want from midwives. However, it is obvious that women's expectations and experiences of midwifery care are of fundamental importance as a research focus.

In order to address the issues around what constitute a good midwife, a thematic analysis about women's perceptions, expectations and understandings of the midwife during labour and birth has been conducted.

What does the childbearing woman value in a midwife? A thematic analysis

On the basis of the rationale previously explained, a thematic analysis was done according to the existing body of knowledge around what childbearing women value in a midwife during labour and birth.

It is clear that women give great importance to the relationship with their midwife as cornerstone of their childbearing event. In particular, the key themes emerging from the thematic analysis were: support, possibility of choice and feeling in control and having appropriate information. Conversely, healthy outcomes, professional competencies and theoretical knowledge seem to be less important to them than to the midwives. Each theme is now discussed in more depth. 


\section{The importance of having a supportive midwife}

Dahlen et al. (2010) state that the caregiver's support seems to strongly influence the relationship between women and midwives. The authors explored first-time mothers' experiences of birth at home and in hospital in Australia using a Grounded Theory methodology. The analysis of data showed that women highly valued the supportive presence of their midwives. In relation to the place of birth, homebirth midwives appeared to be more supportive due to the trusting relationship they established with the woman, their philosophy of care and their freedom from time restrictions and hospital procedures.

The importance of having a supportive midwife has also been underlined by other authors. Berg et al. (1996) undertook a qualitative study in Sweden using a phenomenological approach with the aim of describing 18 women's experience of the encounter with the midwife during childbirth. The authors identified the recurring theme of being supported by the caregiver. Brown et al (Brown et al., 2009) examined the perspectives of women and their partners $(n=10)$ regarding the key roles of the labour and delivery nurse through semi-structured interviews. The caregiver was described as carrying out several important roles, which included support. The participants highlighted the importance of receiving both physical and psychological support. Tumblin \& Simkin (2001) undertook a quantitative study with the objective of determining nulliparous pregnant women's expectations of the labour and delivery nurse's role in a North American setting. The researchers surveyed fifty-seven women, asking them what activities they expected from their nurse during labour and birth. The women listed a total of 174 items. The greatest part of the tasks with a percentage of $29 \%$ was related to providing emotional and informational support. The findings from this study are in contrast with two previous quantitative studies where the authors 
found that labour and delivery nurses spend little time giving women informational support (Gagnon \& Waghorn, 1996; McNiven et al., 1992).

\section{Having choice and feeling in control}

The literature review highlights that most women value making informed choices and feeling in control (Dahlen et al., 2010; Green et al., 1990; Tumblin \& Simkin, 2001). The themes of choice and control seem to be related and their interdependence is supported by Dahlen et al. (2010).

Green et al. (1990) undertook a large-scale prospective questionnaire survey that included 825 women giving birth in six hospitals in England in 1987. The study was designed to assess the relationships between expectations, experiences and psychological outcomes of birth. The data suggest that having appropriate information and feeling in control were important aspects for the woman's well-being during labour and after the birth.

After noting the lack of knowledge around the topic, Green et al. (2000) undertook further research with the aim of examining changes over time (1987-2000) in women's expectations and experiences of intrapartum care and to relate these to psychological outcomes. The authors paid particular attention to the issues of decision-making, choice and control valued by the Changing Childbirth report (Cumberlege et al., 1993). The researchers found that after the Changing Childbirth report some changes in women's expectations occurred, mainly in the area of labour pain and decisionmaking. In fact, women in 2000 were more likely to be anxious about pain in labour and to accept interventions. Green et al. (2000) interpret this as related to the increased use of epidurals and to the diffusion of medicalised models of care. Furthermore, women in 2000 expected and became more involved in non-emergency 
decision making. In fact, they were more satisfied with the information received than women in 1987. Thus, it could be argued that decision-making and possibility of choice are closely associated with the quality and amount of information that the women received from the caregivers.

Women's expectations may also differ by where they choose to give birth. For example, having a predisposition to interventions and epidural may be more important for those who choose hospital. Thus, planned place of birth can be considered an important influencing factor for childbearing women's expectations. However, women's understanding of the midwife's role have not been investigated in relation to different birth places., there is evidence that women giving birth at home or in birth centres have a more positive experience (Waldenstrom \& Nilsson, 1993) and are less likely to regard birth as a medical condition than labour-ward mothers (Cunningham, 1993). Furthermore, Waldenstrom \& Nilsson (1993) argue that birth centre care successfully meets the needs of women who are interested in natural childbirth and active involvement in their own care.

Most women seem to value active involvement in the process of care, the possibility of choice and feeling in control during their childbearing event. However, it must be acknowledged that women sometimes prefer to give professionals the authority to make important decisions, placing themselves in the hands of the caregivers. In fact, some women don't want to makes choices, preferring the midwife or the doctor make it for them (Walker et al., 1995).

The issues around the possibility of choice are connected with the area of moral and ethical values of both the woman and the midwife. The question that arises is whether and how the balance between the midwife's support and the woman's choice/control can be addressed. Kennedy (2000: 12) articulates well an ideal balance, by reporting 
a woman's description about her feeling of achievement in the process of birth: "I've never played football, but if I had, giving birth with her was like catching a winning touchdown in the fourth quarter of a game against a rival, feeling tired and sore, but on top of the world". The key message is that it was the woman who scored the touchdown, not the midwife, but together they were a team that moved toward an identified goal.

\section{The woman values a midwife that gives appropriate information}

Having appropriate information is a precursor to women's possibility of choice. As suggested by Dahlen et al. (2010) the provision of information, communicated clearly by the midwife enables the woman to exercise informed choice. Furthermore, giving appropriate information reduces the woman's fears and enables the childbearing woman to feel more confident in her own potential. Midwives are often described by women and their partners as educators, because 'they are there to provide information, to answer questions, to be somebody who has time to discuss things with the couple about the birthing process' (Brown et al., 2009: 328).

Feeling informed by the midwife was valued by the women $(n=32)$ that gave birth in a midwife-led unit interviewed by Walker et al. (1995). The participants highlighted the importance of having satisfactory information about what is happening or will happen and how to cope with it. Further, they affirm that this is a fundamental condition for personal control and possibility of choice. The greatest majority of the women that delivered in the unit expressed gratitude at being adequately informed at a personal level throughout their labour. For women whose labour did not develop as expected, this was even more critical. However, it is not clear if it the need for information when labour took an unexpected turn was impacted on by a change in the planned place of 
birth. Walsh (2010) states that childbearing women's experiences of birth are often shaped in the uneasy space between the biomedical model and the more "natural" approach of the midwife.

Tumblin \& Simkin (2001) surveyed 57 women aiming at determining nulliparous pregnant women's expectations of their nurse's role during labour and delivery in North America. Giving information/instructions was the key finding. Women seemed to highly value midwives that answered questions $(n=11)$ and helped them with breathing and relaxation techniques. In particular, the results of this study demonstrate that women have clear ideas of the role of the midwife's during labour and birth.

\section{Discussion}

Women's perceptions of what is a good midwife are pivotal because in many countries the midwife is the woman's primary carer and are therefore likely to have a significant impact on whether a mother is satisfied with her birth experience. However, Lewis (1990: 15) argues that 'there has always been a gap between the perceptions and demands of women in respect to maternity policies and practices, and what has been offered by policy-makers and professionals'. Tumblin \& Simkin (2001) claim that fulfilling women's expectations about childbirth can increase women's satisfaction with their birth experiences. Proctor (1998) underlines the importance of understanding the concerns and needs of women by midwives. This is essential in the development of

a woman-centered service in line with current statutory regulation (Nursing \& Midwifery Council, 2008). Furthermore, it has implications for improving the service quality for those who provide and experience the service. 
Since the early 1990's UK government maternity care policy included the aim of providing women with a real possibility of choice regarding the place of birth. To address this, a range of NHS trusts directed initiatives aimed at changing the organisation and delivery of maternity care, moving away from consultant-led care for women with straightforward pregnancy. In this context, midwife-led units and home birth services are becoming increasingly relevant to the configuration of maternity services currently under consideration in UK (Hollowell, 2011). In addition to this, the role of the midwife has undergone a number of changes in recent decades, primarily related to a greater emphasis on woman-centered care.

The literature review reveals information about what is considered to be a good midwife from a range of perspectives and what women value in a midwife, but a dearth of information around women's expectations and experiences specifically of a good midwife, and even less around whether this changes according to where they give birth.

According to the thematic analysis around what childbearing women value in a midwife during labour and birth, great importance is placed on the relationship with their midwife. In particular, frequent key-themes emerging from the literature were: support, possibility of choice, feeling in control and having appropriate information. Establishing a good and trusting rapport may be a necessary condition for quality midwifery practice. Conversely, professional competencies and theoretical knowledge seem to be less important to women. Given the earlier reflections on what regulatory bodies and what midwives themselves say, there appears to be a mismatch between women and midwives.

Furthermore, important changes in practice and in everyday life (e.g. the birth of internet and modern technologies) have taken place in the period of time this literature review refers 
to. This might have caused a significant shift in women's expectations about their childbirth experience. In support to this, Green et al. (2000) found that women's expectations changed in a period of thirteen years, from 1987 to 2000. Thus, the exploration of childbearing women's expectation of the good midwife will add new knowledge to the existing body of literature.

Women's expectations and experiences of the good midwife have not been investigated in relation to different birth settings although this is likely to be an important influencing factor.

In addition to this, most researchers studied women of mixed-parity (nulliparous and multiparous) and few authors considered nulliparous women as a group on their own. However, nulliparous women's experiences are of particular importance as the first birth experience is known to shape future reproductive choices.

\section{Conclusions and implications for practice}

The guiding question of this literature review was: who is a good midwife? A good midwife should possess several attributes: theoretical knowledge, professional competencies, personal qualities, communication skills and moral/ethical values. However, the meaning of good midwife might change according to different actors involved in midwifery care and there is no agreement on the definition of what constitutes a good midwife. Furthermore, it is not clear if what women value in a good midwife corresponds to the midwives' perception of themselves as good professionals. The literature review shows some information about what women value in a midwife, but a dearth of information around nulliparous women's expectations and experiences specifically of a good midwife during childbirth in regard to different birth settings. 
This literature review seeks to stimulate debate and reflection about the nature of the midwife's role, competencies and qualities among midwives and professionals involved in the childbearing event, in order to fulfil women's expectations of their midwife and increase their satisfaction with the birth experience. The identification of the gaps in the evidence provided the starting point and allowed the development of research questions and methodology for an ongoing doctoral research. Based on the gaps in the evidence, the doctoral research will explore and seek to explain nulliparous women's expectations and experiences of a good midwife in the context of different planned place of birth, using a Grounded Theory methodology. It is also expected that the findings of this literature review will stimulate additional research in this area to ultimately inform midwifery practice and midwifery educational programmes. 
Table 1 - Details and critique of the papers included in the thematic analysis

Paper

\section{Research aim}

\section{Sample and setting}

\section{Critique}

\begin{tabular}{llll}
\hline Berg M., Lundgren I., & Describing & 18 women (6 primips, 12 & Q \\
Hermansson E., Wahlberg V. & women's & multips) 2 to 4 days after & res \\
(1996) Women's experience of & experience of the & delivery in the Alternative & P \\
the encounter with the midwife & $\begin{array}{l}\text { encounter with the } \\
\text { Birth Care Centre. }\end{array}$ & in \\
$\begin{array}{ll}\text { during childbirth. Midwifery, 12: } \\
\text { midwife during }\end{array}$ & $\begin{array}{l}\text { SWEDEN. } \\
\text { childbirth. }\end{array}$ & &
\end{tabular}

\section{Qualitative}

research,

interviews.
Phenomenology,

- Clear purpose and background.

- Appropriate methodology and methods.

- Clear audit trail given.

- Clear interpretation of findings and conclusions.

- Demonstration of sensitivity to ethical concerns.

- Limitations of the study and implications for practice outlined.

- Not discussed: philosophical underpinnings, relationship between researcher and participants, researcher's influence on stages of research process, evidence of how problems/complications were dealt with, further investigations.

- The study provides new original insights.

Brown J., Beckhoff J., Stewart M., Examining the Freeman T., Kasperski M. (2009) Women and their partners perceptions of the key roles of the labor and delivery nurse Clinical Nursing Research, 18(4): 323335. perspectives of women and their partners regarding key roles of the

labour and

delivery nurse

during labour and birth.
10 heterosexual couples (women and partners interviewed separately). ENGLAND.

- Clear purpose and background.

research, - Appropriate methodology and methods.

Grounded Theory, - Clear interpretation of findings and conclusions.

interviews.

- Demonstration of sensitivity to ethical concerns.

- Limitations of the study and implications for practice outlined.

- Clear audit trail given.

- Not discussed: philosophical underpinnings, relationship between researcher and participants, researcher's influence on stages of research process, evidence of how problems/complications were dealt with, further investigations.

- The study provides new original insights.

Dahlen H.G., Barclay L.M., Homer C.S.E. (2010) The novice birthing: theorising first-time mothers' experiences of birth at home and in hospital in Australia. Midwifery, 26(1): 53-63.
Exploring firsttime mothers' experiences of birth at home and in hospital in Australia.
19 first-time mothers who gave birth in different birth settings (home, public hospital, private hospital, birth centre) AUSTRALIA
- Clear purpose and background.

Qualitative research,

interviews.

- Clear interpretation of findings and conclusions.

- Demonstration of sensitivity to ethical concerns.

- Implications for practice outlined.

- Clear audit trail given.

- Not discussed: philosophical underpinnings, relationship between researcher and participants, researcher's influence on stages of research process, evidence of how problems/complications were dealt with, limitations of the study, further investigations. 
- $\quad$ The study provides new original insights.

\begin{tabular}{lll}
\hline Green J.M., Coupland V.A., & Determining & $\begin{array}{l}825 \text { women booked for } \\
\text { delivery in } 6 \text { hospitals in }\end{array}$ \\
Kitzinger J.V. (1990) & women's & Southestern England. \\
Expectations, experiences, and & $\begin{array}{l}\text { expectations, } \\
\text { experiences and }\end{array}$ & ENGLAND. \\
$\begin{array}{l}\text { psychological outcomes of } \\
\text { childbirth: a prospective study of } \\
825 \text { women. Birth, 17(1): 15-24. }\end{array}$ & $\begin{array}{l}\text { psychological } \\
\text { outcomes of } \\
\text { childbirth. }\end{array}$ & \\
&
\end{tabular}

\section{Quantitative}

research,

questionnaires.

- Clear purpose and background.

- Appropriate methodology and methods.

- Minimised biases.

- Complete follow up.

- Appropriate statistical analysis.

- Clear interpretation of significant findings and conclusions.

- Demonstration of sensitivity to ethical concerns.

- Limitations of the study and implications for practice outlined.

- Not discussed: further investigations.

- The study provides new original insights.

\section{7 nulliparous women in Quantitative childbirth classes.} Pregnant women's perceptions of their nurse's role during labor and delivery. Birth, 28: 52-56.

\section{Determining}

pregnant women's

expectations of

during labour and

delivery as

expressed during

the last trimester

of pregnancy.
- Clear purpose and background.

research, surveys. - Appropriate methodology and methods.

- Minimised biases.

- Complete follow up.

- Appropriate statistical analysis.

- Clear interpretation of significant findings and conclusions.

- Demonstration of sensitivity to ethical concerns.

- Limitations of the study, implications for practice and further investigations outlined.

- The study provides new original insights.

\section{Walker J.M., Hall S.M., Thomas} M.C. (1995) The experience of labour: a perspective from those receiving care in a midwife-led unit. Midwifery, 11 (3): 120-129.
Elucidate the experience of labour for those receiving any aspect of care in midwife-led unit.
32 women who gave birth in a midwife-led unit and

6 partners during postnatal period. ENGLAND.
Qualitative

research,

Grounded Theory, interviews.
- Clear purpose and background.

- Appropriate methodology and methods.

- Clear interpretation of findings and conclusions.

- Demonstration of sensitivity to ethical concerns

- Limitations of the study and implications for practice outlined.

- Clear audit trail given.

- Not discussed: philosophical underpinnings, relationship between researcher and participants, researcher's influence on stages of research process, evidence of how problems/complications were dealt with, further investigations.

- The study provides new original insights. 


\section{References}

Essential competencies for basic midwifery practice (2010). International Confederation of Midwives.

The code: standards of conduct, performance and ethics for nurses and midwives (2008). Nursing \& Midwifery Council.

Berg M., Lundgren I., Hermansson E., Wahlberg V. (1996) Women's experience of the encounter with the midwife during childbirth. Midwifery, 12: 11-15.

Brown J., Beckhoff J., Stewart M., Freeman T., Kasperski M. (2009) Women and their partners' perceptions of the key roles of the labor and delivery nurse. Clinical Nursing Research, 18(4): 323-335.

Byrom S., Downe S. (2010) "She sort of shines": midwives' accounts of "good" midwifery and "good" leadership. Midwifery, 26: 126-137.

Carolan M. (2010) The good midwife: commencing students' views. Midwifery, 27(4): 503-508.

Carolan M. (2012) 'A good midwife stands out': 3rd year midwifery students' views. Midwifery, doi: 10.1016/j.midw.2011.11.005.

Cumberlege B., Anderson M., Court S., Farmer P., Hutton E., Lightfoot L., Page L., Randhawa K., Troop P., Young G., Mckessack J., Jackson K., Modle J., Shipton J., Houghton G. (1993) Changing Childbirth. Report of the Expert Maternity Group. London: Department of Health.

Cunningham J.D. (1993) Experiences of Australian mothers who gave birth either at home, at a birth centre, or in hospital labour wards. Social Science \&amp; Medicine, 36(4): 475483.

Dahlen H.G., Barclay L.M., Homer C.S.E. (2010) The novice birthing: theorising first-time mothers' experiences of birth at home and in hospital in Australia. Midwifery, 26(1): 5363.

Eliasson M., Kainz G., Von-Post I. (2008) Uncaring midwives. Nursing Ethics, 15(4): 500-511.

Fontein Y. (2009) Midwifery care in the community. In: Mander, R., Fleming V. Becoming a midwife. Oxon: Routledge.

Freeman L.M., Timperley H., Adair V. (2004) Partnership in midwifery care in New Zealand. Midwifery, 20(1): 2-14.

Fullerton J., Thompson J., Severino R. (2011) The International Confederation of Midwives essential competencies for basic midwifery practice. Midwifery, 27: 399-408.

Gagnon A., Waghorn K. (1996) Supportive care by maternity nurses: a work sampling study in an intrapartum unit. Birth, 23(1): 1-6.

Green J., Baston H., Easton S., Mccormick F. (2000) Greater expectations? A comparison of women's expectations and experiences of maternity care choices in 1987 and 2000.

Green J.M., Coupland V.A., Kitzinger J.V. (1990) Expectations, experiences, and psychological outcomes of childbirth: a prospective study of 825 women. Birth, 17(1): 15-24.

Halldorsdottir S., Karlsdottir S. (2011) The primacy of the good midwife in midwifery services: an evolving theory of professionalism in midwifery. Scandinavian Journal of Caring Sciences.

Halldorsdottir S., Karlsdottir S.I. (1996) Empowerment or discouragement: women's experience of caring and uncaring encounters during childbirth. Health Care for Women International, 17(4): 361-379.

Hollowell J. (2011) Birthplace programme overview: background, component studies and summary of findings. Birthplace in England research programme. Final report part 1. NIHR Service Delivery and Organisation programme.

Kennedy H. (2000) A model of exemplary midwifery practice: results of a Delphy study. Journal of Midwifery \& Women's Health, 45(5): 4-19. 
Larkin P., Begley C.M., Devane D. (2012) 'Not enough people to look after you': an exploration of women's experiences of childbirth in the Republic of Ireland. Midwifery, 28(1): 98105.

Leap N. (2000) The less we do the more we give. In: Kirkham, M. The midwife-mother relationship. London: McMillan.

Lewis J. (1990) Mothers and maternity policies in the twentieth century. In: Garcia, J., Kilpatrick R., Richards M. The politics of maternity care. Services for childbearing women in twentieth-century Britain. Oxford: Clarendon Press.

Mcniven P., Hodnett E., O'brien-Pallas L.L. (1992) Supporting women in labor: a work sampling study of the activities of labor and delivery nurses. Birth, 19(1): 3-8.

Nicholls L., Skirton H., Webb C. (2011) Establishing perceptions of a good midwife: a Delphi study. British Journal of Midwifery, 19(4): 230-236.

Nicholls L., Webb C. (2006) What makes a good midwife? An integrative review of methodologically-diverse research. Journal of Advanced Nursing, 56(4): 414-429.

Pembroke N.F., Pembroke J.J. (2008) The spirituality of presence in midwifery care. Midwifery, 24(3): 321-327.

Proctor S. (1998) What determines quality in maternity care? Comparing the perceptions of childbearing women and midwives. Birth, 25(2): 85-93.

Sellman D. (2007) On being of good character: nurse education and the assessment of good character. Nurse Education Today, 27(7): 762-7.

Simkin P. (1991) Just another day in a woman's life? Women's long-term perceptions of their first birth experience. Birth, 18(4): 203-210.

Tumblin A., Simkin P. (2001) Pregnant women's perceptions of their nurse's role during labor and delivery. Birth, 28(1): 52-56.

Waldenstrom U. (1998) Continuity of carer and satisfaction. Midwifery, 14: 207-213.

Waldenstrom U., Nilsson M.I. (1993) Women's satisfaction with birth center care: a randomized, controlled study. Birth, 20(1): 3-13.

Walker J.M., Hall S.M., Thomas M.C. (1995) The experience of labour: a perspective from those receiving care in a midwife-led unit. Midwifery, 11(3): 120-129.

Walsh D.J. (2010) Childbirth embodiment: problematic aspects of current understandings. Sociology of Health \& IIIness, 32(3): 486-501. 